\title{
Consistency Conditions for Difference Schemes with Singular Coefficients*
}

\author{
By Dennis Eisen
}

I. Introduction. In [1], the author considered the stability and convergence of finite-difference approximations to a certain class of partial differential equations containing singular coefficients. It was found, in particular, that straightforward replacement of derivatives by corresponding difference quotients could often lead to difference operators which were unbounded, even with respect to the $L_{2}$ norm. This negated any chance of stability, and by the Lax Equivalence Theorem [2], of convergence as well.

One way out of this difficulty, as discussed in [1], is to measure stability and convergence with respect to the sequence of mean $p$ th power norms

$$
\|U\|_{M_{p}}=\left\{\frac{1}{M} \sum_{j=1}^{M}\left|U_{j}\right|^{P}\right\}^{1 / P}, \quad 1 \leqq p \leqq \infty,
$$

where $M=M(\Delta t)$ is the number of lattice points of the finite-difference grid.

It is the purpose of the present paper to note that the same sort of troubles can occur when investigating the consistency of an approximation to an initial-value problem containing singular coefficients. That is, straightforward replacement of derivatives by difference quotients may often result in schemes which are not consistent in the usual Lax-Richtmyer sense. As an example of this, consider the $m$ dimensional, spherically symmetric diffusion equation

$$
\frac{\partial u}{\partial t}=\frac{\partial^{2} u}{\partial r^{2}}+\frac{(m-1)}{r} \frac{\partial u}{\partial r}, \quad 0 \leqq t \leqq T,
$$

with initial and regularity conditions of the form

$$
u(r, 0)=u_{0}(r) \quad \text { and } \quad \partial u(0, t) / \partial r=0 .
$$

The spatial domain is an $m$-sphere of radius $R$, but the precise nature of the boundary conditions there need not concern us. A simple approximation to (1.2) is given by

$$
\begin{aligned}
v(r, t+\Delta t)= & v(r, t)+\lambda[v(r+\Delta r, t)-2 v(r, t)+v(r-\Delta r, t)] \\
& +((m-1) / r)(\lambda \Delta t)^{1 / 2}[v(r+\Delta r, t)-v(r, t)] \\
\equiv & C(\Delta t) v(r, t)
\end{aligned}
$$

where $\lambda=\Delta t /(\Delta r)^{2}$. We take $v(r, 0)=u_{0}(r)$ and utilize the regularity condition to specify that $v(\Delta r-r, t)=v(r-\Delta r, t)$ when $r-\Delta r<0$.

According to the Lax-Richtmyer theory, if (1.4) is to be consistent with the

Received January 16, 1967 .

* This work performed under the auspices of the U. S. Atomic Energy Commission. 
differential equation (1.2) we must have

$$
\left\|\left\{\frac{C(\Delta t)-I}{\Delta t}-\frac{\partial}{\partial t}\right\} u(r, t)\right\| \rightarrow 0 \text { as } \Delta t \rightarrow 0
$$

uniformly in $t \in[0, T]$, for a sufficiently wide class of genuine solutions. Substitution for $C(\Delta t)$ and $u_{t}$ from (1.4) and (1.2), respectively, will show that

$$
\begin{aligned}
\left\{\frac{C(\Delta t)-I}{\Delta t}-\frac{\partial}{\partial t}\right\} u(r, t)= & \frac{(\Delta r)^{2}}{12} \frac{\partial^{4} u}{\partial r^{4}}+\frac{(m-1)}{2 r} \Delta r \frac{\partial^{2} u}{\partial r^{2}} \\
& +O\left[(\Delta r)^{4}\right]+O\left[\frac{(\Delta r)^{2}}{r}\right]
\end{aligned}
$$

if $u(r, t)$ is smooth enough, and utilizing the $L_{2}$ norm shows that

$$
\begin{aligned}
\left\|\left\{\frac{C(\Delta t)-I}{\Delta t}-\frac{\partial}{\partial t}\right\} u(r, t)\right\|^{2}= & \int_{0}^{R}\left[\frac{(m-1)}{2 r} \Delta r \frac{\partial^{2} u}{\partial r^{2}}\right]^{2} d r \\
& + \text { remaining terms } .
\end{aligned}
$$

It is well known (see [3], for example) that the genuine solutions of (1.2) are analytic for $t>0$ and are such that lim inf $u_{r r}>0$ as $r \rightarrow 0$. This means that the function $\Delta r \cdot u_{r r} / r$ appearing in (1.6) will not be in $L_{2}$ for any $\Delta r \neq 0$, and it follows that condition (1.5) cannot possibly be fulfilled. We therefore have

Lемма 1. The difference approximation (1.4) is not consistent with the differential equation (1.2) when measured with respect to the $L_{2}$ norm.

We do not, however, wish to abandon (1.4) and we shall therefore investigate consistency with respect to the mean $p$ th power norm (1.1).

II. Sufficient Conditions for Mean $p$ th Power Consistency. Let us consider a general initial-value problem of the form $u(0)=u_{0}$ and

$$
u_{t}=A u=\sum_{\nu=0}^{N} \sum_{k_{1}+k_{2}+\cdots+k_{Q}=\nu} A_{k_{1} k_{2} \cdots k_{Q}} \frac{\partial^{\nu} u}{\partial x_{1}{ }^{k_{1}} \partial x_{2}^{k_{2}} \cdots \partial x_{Q}{ }^{k_{Q}}},
$$

where the $A_{k_{1} k_{2} \cdots k_{Q}}$ are functions of $x$, and $u$ is a $P$ component vector.

The corresponding finite-difference operator $C(\Delta t)$ must take the form

$$
C(\Delta t)=\sum_{k_{1}} \sum_{k_{2}} \cdots \sum_{k_{Q}} c_{k_{1} k_{2} \cdots k_{Q}} T\left(k_{1} \Delta x_{1}, k_{2} \Delta x_{2}, \cdots, k_{Q} \Delta x_{Q}\right)
$$

where the $c_{k_{1} k_{2} \cdots k_{Q}}$ are $P \times P$ matrices and $T$ denotes the translation operator. Since

$$
\begin{aligned}
T\left(k_{1} \Delta x_{1},\right. & \left.k_{2} \Delta x_{2}, \cdots, k_{Q} \Delta x_{Q}\right) \\
& =\sum_{\nu=0}^{\infty} \frac{1}{\nu !}\left[k_{1} \Delta x_{1} \frac{\partial}{\partial x_{1}}+k_{2} \Delta x_{2} \frac{\partial}{\partial x_{2}}+\cdots+k_{Q} \Delta x_{Q} \frac{\partial}{\partial x_{Q}}\right]^{\nu} \\
& =\sum_{\nu=0}^{\infty} \frac{1}{\nu !}\left\{\sum_{l_{1}+l_{2}+\cdots+l_{Q}=\nu} \frac{\nu !\left(k_{1} \Delta x_{1}\right)^{l_{1}}\left(k_{2} \Delta x_{2}\right)^{l_{2}} \cdots\left(k_{Q} \Delta x_{Q}\right)^{l_{Q}}}{\left(l_{1}\right) !\left(l_{2}\right) ! \cdots\left(l_{Q}\right) !} \frac{\partial^{\nu}}{\partial x_{1}{ }^{l_{1}} \partial x_{2}{ }^{l_{2}} \cdots \partial x_{Q}{ }^{l_{Q}}}\right\}
\end{aligned}
$$

by the multinomial expansion, we may therefore write 


$$
\begin{aligned}
C(\Delta t)= & \sum_{\nu=0}^{\infty} \sum_{l_{1}+\cdots+l_{Q=\nu}} \sum_{k_{1}} \cdots \sum_{k_{Q}} \frac{\left(k_{1} \Delta x_{1}\right)^{l_{1}} \cdots\left(k_{Q} \Delta x_{Q}\right)^{l_{Q}}}{\left(l_{1}\right) ! \cdots\left(l_{Q}\right) !} \\
& \cdot c_{k_{1} \cdots k_{Q}} \frac{\partial^{\nu}}{\partial x_{1}^{l_{1}} \cdots \partial x_{Q}{ }^{l_{Q}}},
\end{aligned}
$$

where the interchange in summation is certainly valid for explicit schemes and for all implicit schemes where $c_{k_{1} k_{2} \cdots k_{Q}} \rightarrow 0$ fast enough as any of the $k_{i} \rightarrow \pm \infty$.

Substituting (2.1) and (2.3) into the consistency relation (1.5), we obtain for a sufficiently smooth set of genuine solutions

$$
\begin{gathered}
\left\|\left\{\frac{C(\Delta t)-I}{(\Delta t)}-A\right\} u(t)\right\|_{M_{p}} \\
=\| \sum_{\nu=0}^{\infty} \sum_{l_{1}+\cdots+l_{Q}=\nu}\left\{-\frac{I \delta_{\nu_{0}}}{\Delta t}+\frac{1}{\Delta t} \sum_{k_{1}} \cdots \sum_{k_{Q}} \frac{\left(k_{1} \Delta x_{1}\right)^{l_{1}} \cdots\left(k_{Q} \Delta x_{Q}\right)^{l_{Q}}}{\left(l_{1}\right) ! \cdots\left(l_{Q}\right) !}\right. \\
\left.\cdot c_{k_{1} \cdots k_{Q}}-A_{l_{1} \cdots l_{Q}}\right\} \cdot \frac{\partial^{\nu} u}{\partial x_{1}{ }^{l_{1}} \cdots x_{Q}{ }^{l_{Q}}} \|_{M_{p}}
\end{gathered}
$$

where we define $A_{l_{1} \cdots l_{Q}} \equiv 0$ if $l_{1}+\cdots+l_{Q}>N$, and where $\delta_{\nu 0}$ is the Kronecker delta $\left(\delta_{\nu 0}=1\right.$ if $\nu=0$, zero otherwise). Choosing some integer $N^{*} \geqq N$, and using the triangle inequality,

$$
\begin{aligned}
& \left\|\left\{\frac{C(\Delta t)-I}{\Delta t}-A\right\} u(t)\right\|_{M_{p}} \leqq \sum_{\nu=0}^{N^{*}} \sum_{l_{1}+\cdots+l_{Q=\nu}} \\
& \left\|\left\{-\frac{I \delta_{\nu 0}}{\Delta t}+\frac{1}{\Delta t} \sum_{k_{1}} \cdots \sum_{k_{Q}} \frac{\left(k_{1} \Delta x_{1}\right)^{l_{1}} \cdots\left(k_{Q} \Delta x_{Q}\right)^{l_{Q}}}{\left(l_{1}\right) ! \cdots\left(l_{Q}\right) !} c_{k_{1} \cdots k_{Q}}-A_{l_{1} \cdots l_{Q}}\right\}\right\|_{M} \\
& \cdot\left\|\frac{\partial^{\nu} u}{\partial x_{1}^{l_{1}} \cdots \partial x_{Q}{ }^{l} Q}\right\|_{M_{p}}+\sum_{l_{1}+\cdots+l_{Q}=N^{*}+1} \\
& \left\|\frac{1}{\Delta t} \sum_{k_{1}} \cdots \sum_{k_{Q}} \frac{\left(k_{1} \Delta x_{1}\right)^{l_{1}} \cdots\left(k_{Q} \Delta x_{Q}\right)^{l_{Q}}}{\left(l_{1}\right) ! \cdots\left(l_{Q}\right) !} c_{k_{1} \cdots k_{Q}} \frac{\partial^{N^{*}+1} u(\xi)}{\partial x_{1}{ }^{l_{1}} \cdots \partial x_{Q}{ }^{l_{Q}}}\right\|_{M_{p}},
\end{aligned}
$$

where $\xi$ is a $Q$-component vector which lies along the line between the point $\left(x_{1}, x_{2}, \cdots, x_{Q}\right)$ and $\left(x_{1}+k_{1} \Delta x_{1}, x_{2}+k_{2} \Delta x_{2}, \cdots, x_{Q}+k_{Q} \Delta x_{Q}\right)$.

The expression (2.4) permits us to state the

Theorem. For a well-posed initial-value problem of the form (2.1), suppose for some integer $N^{*} \geqq N$ we have the two conditions

$$
\begin{gathered}
\lim _{\Delta t \rightarrow 0} \|\left\{-\frac{I \delta_{\nu 0}}{\Delta t}+\frac{1}{\Delta t} \sum_{k_{1}} \cdots \sum_{k_{Q}} \frac{\left(k_{1} \Delta x_{1}\right)^{l_{1}} \cdots\left(k_{Q} \Delta x_{Q}\right)^{l_{Q}}}{\left(l_{1}\right) ! \cdots\left(l_{Q}\right) !}\right. \\
\left.\cdot c_{k_{1} \cdots k_{Q}}-A_{l_{1} \cdots l_{Q}}\right\} \|_{M_{p}}=0
\end{gathered}
$$

for all $l_{i} \geqq 0$ such that $l_{1}+l_{2}+\cdots+l_{Q}=\nu$ and $\nu=0,1,2, \cdots, N^{*}$, where $A_{l_{1} \cdots l_{Q}}=0$ if $l_{1}+\cdots+l_{Q}>N$, 


$$
\begin{aligned}
& \lim _{\Delta t \rightarrow 0}\left\{\frac{\left(\Delta x_{1}\right)^{l_{1}} \cdot\left(\Delta x_{2}\right)^{l_{2}} \cdots\left(\Delta x_{Q}\right)^{l_{Q}}}{\Delta t}\right\}=0 \\
& \text { for all } l_{1}+l_{2}+\cdots+l_{Q}=N^{*}+1 .
\end{aligned}
$$

Then the finite-difference operator (2.2 must be consistent with the initial-value problem (2.1) when measured with respect to the mean pth power norm.

III. An Application. As an example consider the difference scheme (1.4) for the diffusion equation. In this case $Q=P=1$ and we have

$$
\begin{aligned}
A_{0}= & 0, \quad A_{1}=(m-1) / r, \quad A_{2}=1, \\
c_{-1}(j \Delta r)= & \lambda, \quad c_{0}(j \Delta r)=1-2 \lambda-(m-1) \lambda / j, \\
& c_{+1}(j \Delta r)=(1+(m-1) / j) \lambda,
\end{aligned}
$$

where the difference scheme is defined on the lattice $r=j \Delta r$ and $j=$ $j_{0}, j_{0}+1, \cdots, j_{0}+M-1$ for $0<j_{0}<1$. Then with (3.1) the sums in (2.5) become,

$$
\text { for } \begin{aligned}
\nu & =0: \frac{-1}{\Delta t}+\frac{1}{\Delta t}\left\{c_{-1}(j \Delta r)+c_{0}(j \Delta r)+c_{+1}(j \Delta r)\right\}=0, \\
\nu & =1: \frac{\Delta r}{\Delta t}\left\{-c_{-1}(j \Delta r)+c_{+1}(j \Delta r)\right\}-A_{1}(j \Delta r)=0, \\
\nu & =N=2: \frac{(\Delta r)^{2}}{2 \Delta t}\left\{c_{-1}(j \Delta r)+c_{+1}(j \Delta r)\right\}-A_{2}(j \Delta r)=\frac{m-1}{2 j} .
\end{aligned}
$$

The second condition (2.6) is fulfilled because $\lim _{\Delta t \rightarrow 0}(\Delta r)^{3} / \Delta t=0$, and we must therefore investigate the mean $p$ th power norm of the vector $\{(m-1) / 2 j\}$, $j=j_{0}, j_{0}+1, \cdots, j_{0}+M-1$. We cannot take $p=\infty$, for the $M_{\infty}$ norm of the vector $\{1 / j\}$ is simply $1 / j_{0}$, but any finite value of $p$ will do. For example, if $p=1$, then

$$
\left\|\left\{\frac{1}{j}\right\}\right\|_{M_{1}}=\left\{\frac{1}{M} \sum_{j=1}^{M}\left|\frac{1}{j_{0}+j-1}\right|\right\} \sim \frac{\ln M}{M} \rightarrow 0 \text { as } \Delta t \rightarrow 0,
$$

and in general

$$
\begin{aligned}
\left\|\left\{\frac{1}{j}\right\}\right\|_{M_{p}}=\left\{\frac{1}{M} \sum_{j=1}^{M}\left|\frac{1}{j_{0}+j-1}\right|^{p}\right\}^{1 / p}=\frac{1}{M^{1 / p}} O(1) \rightarrow 0 \\
\text { as } \Delta t \rightarrow 0, \quad 1<p<\infty .
\end{aligned}
$$

We have therefore established

Lemma 2. The difference scheme (1.4) is a consistent approximation to the diffusion equation (1.2) with respect to any mean pth power norm, $1 \leqq p<\infty$.

Establishing convergence of (1.4) in the $M_{p}$ norm is quite another matter, however. If we select midpoint spacing of the finite-difference grid (i.e. $j_{0}=\frac{1}{2}$ ), then the regularity condition (1.3) is enforced by the symmetry condition $v(\Delta r / 2, t)$ $=v(-\Delta r / 2, t)$, and the corresponding difference matrices will take the form 


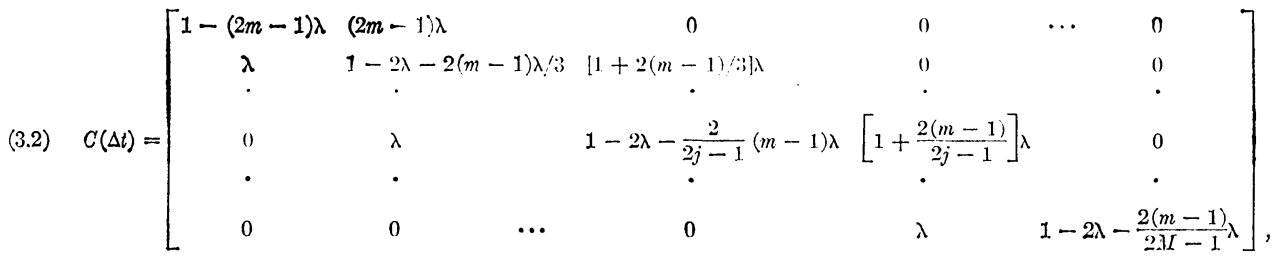

if it is assumed, for example, that $u(R, t)=0$. We note that when $0 \leqq \lambda \leqq$ $1 /(2 m-1)$ and $m \geqq 2$ all elements in (3.2) are nonnegative and therefore

$$
\|C\|_{M_{\infty}}=\max _{(i)}\left\{\sum_{j=1}^{M}\left|C_{i j}\right|\right\}=1 \text {. }
$$

This yields

Lemma 3. The difference scheme (1.4) for $m \geqq 2$ with midpoint spacing is stable in the $M_{\infty}$ norm if $0 \leqq \lambda \leqq 1 /(2 m-1)$.

Unfortunately, we do not have the requisite consistency in the $M_{\infty}$ norm so as to be able to conclude the convergence of (1.4). As (1.2)-(1.3) is well posed, all we can say for now is that if $E(t)$ denotes the solution operator, then

$$
\left\|\left\{C^{n}(\Delta t)-E(t)\right\} u_{0}\right\|_{M_{p}} \leqq\left\|\left\{C^{n}(\Delta t)-E(t)\right\} u_{0}\right\|_{M_{\infty}}=O(1)
$$

as $\Delta t \rightarrow 0$ and $n \Delta t \rightarrow t$ for any continuous initial function $u_{0}$.

Applied Mathematics Department

Brookhaven National Laboratory

Upton, L. I., New York

1. D. EISEN, "Stability and convergence of finite difference schemes with singular coefficients," SI AM J. Numer. Anal., v. 4, 1966, no. 4.

2. R. D. Richtmyer, Difference Methods for Initial Value Problems, Interscience, New York, 1957. MR $22 \# 3864$.

3. G. Hellwig, Partial Differential Equations, Blaisdell, New York, 1964. MR 30 \#3286. 